\title{
A Tailored Approach is Key: the Health Guardian for Longevity Program Uses Mobile Technology to Sustain Healthy Life Behaviors
}

\author{
Freida Pemberton* \\ Molloy College, USA \\ *Corresponding author: Freida Pemberton, Full Professor of Nursing, Molloy College, Barbara H Hagan Center for Nursing Building-Office 217, USA
}

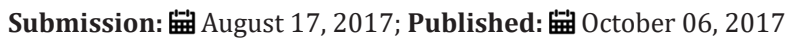

\begin{abstract}
The purpose of this article is to generate interest and excitement in an innovative approach to patient-centered, custom-tailored health care that is offered by the Health Guardian for Longevity program. The Health Guardian for Longevity program reflects an assimilation of the principals of nursing informatics and integrates the sciences of nursing, information, and computer, designed to improve the quality of care and sustain healthy life-style behaviors that incorporate the individual, family, and community uniqueness. The Health Guardian for Longevity program takes in hand the client's assessed data, while activating the powerful tools of mobile, interactive, remote, video, and virtual technologies, as well as selected learning models and behavioral theories.

The ultimate goal of this construct is to provide tailored centered patient care that addresses the holistic needs of the individual. In addition, the program focus is on initiatives that have the potential to enhance the opportunity for patients to become effective self-managers of care. This multifaceted approach to health care allows caregivers and their patients to focus on the patient's unique needs to restore, maintain, and integrate healthier behavioral practices. In conclusion, the author illustrates the application of the Health Guardian for Longevity Program and highlights plans for implementation both nationally and internationally. A case study of the impact of the Health Guardian for Longevity Program on sustainability of healthy life-style behaviors is forthcoming.
\end{abstract}

\section{Introduction}

This presentation is designed to generate interest and excitement in the innovative approach to patient-centered, customtailored health promotion offered by the Health Guardian for Longevity program. The Health Guardian for Longevity program takes in hand the assessed data, mobilizing the powerful tools of mobile, interactive, remote, video, and virtual technologies, as well as selected learning models, behavioral theories, and humor to treat patients as individuals. This multifaceted approach allows caregivers and their patients to focus on the patient's unique needs to restore, maintain, and integrate healthier behavioral practices.

The primary motivation for the development of the program and the program's key objective - is to get individuals more directly involved in their own recovery and self-care after diagnosis or crisis. This objective can be achieved by cultivating an environment of convenience, personalization and comfort in which the health care consumer is not only a patient but a client. The Health Guardian for Longevity program empowers individuals to become effective advance managers of their health care, speeding the transition from unwell to well and achieving better long-term outcomes.

Kreuter [1] defined tailoring as "Any combination of strategies and information intended to reach one specific person, based on characteristics that are unique to that person, related to the outcome of interest and derived from an individual assessment". In their report on information retention [2], Pennsylvania State University's Task Force on Distance Education noted that individuals retain approximately $20 \%$ of information transmitted by verbal means alone, and $40 \%$ of what is transmitted only visually. However, when one transmits information through a combination of verbal, visual, and hands-on media, the retention rate surges to approximately $75 \%$.

Using Kreuter [1] definition of tailoring, and the Penn State task force's report as departure points, we expect that the personalized, interactive media used in the development of the Health Guardian for Longevity program will result in markedly improved information retention and utilization on the part of patient-clients using the program. The varied telecommunication technology integrated in the Health Guardian for Longevity Program better addresses the individuality and unique healthcare needs of the patient-client while maintaining their interest and enhancing their motivation to practice healthy lifestyle behaviors.

\section{Background}

In reviewing the Vital Statistics Data of the New York State Department of Health-Bureau of Biometrics and Health Statistics [3], it was alarming to learn that in 2009 alone, 144,860 lives were 
lost as a result of heart disease, cancer, chronic lower respiratory disease (CLRD), stroke, pneumonia and influenza. According to the February 2011 report, the distribution of deaths for the 2009 year was as follows:

Heart disease-46,311, cancer-34,820, CLRD-6,661, stroke 5,823 , pneumonia and influenza-4,383. The ailments behind these statistics continue to challenge health care providers to produce innovative solutions that will not only resolve their patient-clients' existing health problems, but reduce their future occurrences.

Skinner [4], note the increasing body of evidence indicating tailored patient-centered health education programs that are based on detailed background and cultural information, individualize care, particular health behaviors, beliefs and practices yield improved outcomes. The consensus of research by Gustafson, Hawkins, Boberg [5] supports the assertion that the better the patient-client's understanding of their own diagnosis, treatment, and recovery plan - and the better his or her support system - the better that patientclient can cope with an illness and adopt behaviors more likely to facilitate both a positive short-term outcome as well as long-term health.

The research also suggests that patients recovering in Mobile Home Healthcare Environments have a decrease in mortality rates, largely due to improved, more consistent self-care behaviors. Kreuter [1] pointed out, as research continues to show tailored interventions to be more effective than traditional approaches to health education and communication, more health care providers may consider using this approach to enhance outcomes for their own patient-clients. The Health Guardian for Longevity program represents a powerful new tool in providers' arsenal, and we look forward to shaping research on the impact it has on patient-client outcomes when compared with more traditional, one-size-fits-all interventions.

The synthesis of the information before us was extremely motivating and inspired us to create a program that encompassed many of the elements we found missing in more traditional interventions, as well as taking full advantage of current technology, including Bluetooth, remote interactive terminals, video conferencing, customized applications and web pages, and 3G/4G smart phones, with the appropriate technology being utilized to meet each individual patient-client's needs and capabilities.

\section{Evolution of the Health Guardian for Longevity Program}

Long before any research for this project began however, the fundamental aim of the Health Guardian for Longevity program was inspired by the deaths of several family members, friends and associates at early ages from a number of the ailments referenced earlier in the New York State Department of Health report. It seemed that the more traditional, generalist practices being applied to these patients consistently failed to address both the holistic and the specific needs of the individual, ignoring-or at least overlookingcultural practices, spirituality, education level, stress levels, and beliefs about health care practices and behaviors integral to the patient-client's unique profile.
Realizing that historically, attempts to create educational tailored, patient-centered health promotion programs have been limited, we were energized to integrate the sciences of information and computer technology, social behavior and nursing to develop the Health Guardian for Longevity program. The overall design of the program takes into account face-to-face interaction, remote monitoring, virtual and remote access, video conferencing, video personalization, and humor to empower patient-clients to take the lead in all aspects of their health care and address their own specific needs. Our focus on interactive mobile technology addresses the ubiquity of smart phones, tablet computers, and other personal data devices across all demographics. The Health Guardian for Longevity program integrates these concepts and outreach methods to achieve the following:

1. Empower the patient-client in their home environment

2. Enhance their understanding of their disease, treatment, recovery and maintenance

\section{Enhance the patient-client's coping skills}

4. Facilitate healthy behaviors holistically, with humor and personal connection

The cornerstone of the program is the unique, customizable web page constructed for each program participant. Upon release from the hospital or care facility, the patient-client is equipped with both the hardware and software needed to participate. Depending on the participant's needs and capabilities, a typical home setup might include a loaner laptop with wireless technology for Internet access and equipped with a webcam; a Smartphone with 4G capability pre-loaded with remote monitoring functions and customized interactive apps, and other peripherals as needed. The patientclient is also provided a password to access their private Health Guardian web page. This page will have been designed to address their specific needs, and incorporates meaningful background, sound and graphics related to their cultural practices, beliefs and areas of expertise.

Navigation through the website and personal pages is intuitive and user-friendly to optimize the patient-client's comfort with the technology. The Health Guardian for Longevity homepage opens with a welcome video that presents an overview of the main site and gives users information on how to utilize their personal page to manage their care and improve their health status. Program participants use a simple set of Navigation Buttons to move around the Program homepage and their own personal page.

The Health Guardian Welcome Button activates a personal message from the patient-client's caregiver - their 'Health Guardian Partner'-welcoming them back home, and providing an in-depth explanation of the events that led to the patient-client's hospitalization or state of crisis, and providing an update of their status upon discharge. The Welcome screen can even obtain the participant's vital signs via remote monitoring, and explain the program that has been created specifically for him or her.

Recognizing that information is power and that few laypeople possess detailed knowledge of anatomy and the functions of 
various body systems, the Interactive Anatomy Button enables the patient-client to connect via app to an interactive module program explaining the normal Functioning of the body system or systems affected by their recent diagnosis or procedure. The patient is able to progress through the program at their own pace. This feature allows the participant to gain a much better understanding of the alteration that may have occurred as a result of their health crisis, and enables them to become more conversant with their own care providers as their treatment and aftercare progress. For patientclients wishing even more detailed information, the Theories Button serves as a resource for the patient to have greater understanding of the causative factors related to the disease process or illness.

To help address what is often a problematic lack of face time between providers and patient-clients, the Interactive Actual Disease Explanation Button enables the participant's Health Guardian Partner to discuss more in-depth the causative factors that led to the patient's illness, diagnosis, and/or hospitalization. The Health Guardian Partner will share actual theories to explain these causative factors and document this information on the web page using text associations and graphics reflective of their area of expertise. This additional information enhances meaning for the patient-client, giving them greater insight into the process and increasing their compliance as a result. In addition, the patient will be connected via app to an interactive module program that explains their condition (i.e., Coronary Heart Disease) and its impact on the affected body system(s). The patient will have a better understanding of the clinical manifestations they experienced, and the information they gain will hopefully encourage them to adopt the regimen recommended to them for improved future health. For patient-clients wanting even more in-depth information about their condition, the Theories Button serves as a resource for greater understanding of the causative factors related to the illness or disease process.

Inspired by our years of hands-on nursing experience, the Intervention Button provides program participants with specific, targeted interventions to resolve their illness and restore them to a point of stability. The patient-client's personal interventions will be discussed further in face-to-face sessions with his or her caregivers using voice or video conferencing (i.e. Skype, Face Time, etc.). Should a situation arise that requires immediate attention, the patient-client has 24/7 access to their Health Guardian Partner via the Video Conference Call Button.

Links to other resources of interest or use to the patient-client to enhance their knowledge and be reflective of their progression will be accessible via the Website Button. Resources and items of interest will be periodically updated as the program participant recuperates and his or her needs and priorities evolve.

And finally, because we firmly believe that 'laughter is the best medicine,' the program participant will have access to the Humor Button, linking them to a brief, humorous video related to their specific health issue and posted to their personal web page or to YouTube. A preview of the website's functionality can be seen at www.healthguardianforlongevity.com. To see the humor video, just click on the Humor Button, or go to YouTube and search "Health Guardian." As illustration of the navigation layout described, the home page is shown below.

\section{Illustration: Home Page}

In developing this program, we were mindful that the motivation of the patient-client is central in reducing risk, increasing compliance and reducing the likelihood of return trips to the health care facility - the dreaded 'revolving door syndrome. DiMarco [6] encouraged health care providers to improve patient-client motivation by incorporating technology in creating individualized patient programs. The fusion of personal attention and easy, pointand-click access to health information helps patient-clients better understand and make more informed decisions about their care.

\section{Application of the Health Guardian for Longevity Program}

The Health Guardian for Longevity program prototype was tested first internally on the Health Guardian Partners. Following that, it was rolled out to a cluster of healthcare consumers in the communities of Nassau and Queens for external testing.

Initially, we used the program to model various "what if" scenarios, had our own late loved ones and associates had the opportunity to experience the Health Guardian for Longevity Program after their diagnoses and during their various treatments. Prior to being rolled out to actual health care consumers, the Health Guardian for Longevity program was run using more detailed virtual case scenarios. One such virtual scenario was that of Ms. C. Ms. C. is a 40-year-old woman who has worked as a high-level executive accountant for the past two years. She is originally from Ghana, West Africa and has been a U.S. citizen for the past five years. She has three teenage children: a 17-year-old son, a 15-year-old son with a disability, and 13-year-old daughter. After 20 years of marriage, she is currently in the process of a challenging divorce. Ms. C. stands 5'4" and currently weighs 130 lbs. Her medical history is unremarkable, with no family history of heart disease.

Last week, Ms. C. was hospitalized after suffering a heart attack and is currently being discharged. In addition to the usual prescriptions and post-discharge instructions, Ms. C. has been enrolled in the Health Guardian for Longevity program, and equipped with the tools Needed to participate. Her home setup consists of a loaner laptop with a webcam, built-in speakers, and Internet access; she is also given a smartphone with 4G capability, pre-loaded with remote monitoring functions and interactive applications customized to meet her specific needs. Ms. C. is provided an access code that enables her to gain entry to her own private page on the Health Guardian for Longevity website, which was created for her.

The illustration (Table 1 and Figure 1) below depicts the interwoven concepts and technology constructs incorporated in the tailored Health Guardian for Longevity Program designed for Ms. C., determined by her assessed data. Early implementations of the Health Guardian for Longevity program prototype strongly 
suggest that the program has the potential to effect positive change in patient-client health outcomes-not only locally, but globally. Live patient-client participants who have used the program thus far have expressed a personal connection to the process, and identified the intervention as being special to them.

Table 1: Interwoven concepts and technology constructs for Ms. C.

\begin{tabular}{|c|c|c|c|c|}
\hline Problem & Critical Data & Theory Explanation & Intervention: Stress Reduction & Technology \\
\hline \multirow[t]{7}{*}{ Heart Attack } & $\begin{array}{l}\text { High-Level } \\
\text { Executive }\end{array}$ & Normal Heart & Assert Yourself & $\begin{array}{c}\text { Laptop, Smart Phone, Webcam, Internet Access, Virtual } \\
\text { individual Sub-website }\end{array}$ \\
\hline & $\begin{array}{l}\text { Challenge of } \\
\text { Divorce }\end{array}$ & Heart Attack & Regular Aerobic Exercise & $\begin{array}{c}\text { Online Apps Interactive Module I and II Remote } \\
\text { Monitoring Technology }\end{array}$ \\
\hline & $\begin{array}{c}\text { 3Teenagers } 1 \\
\text { Child with a } \\
\text { disability }\end{array}$ & $\begin{array}{l}\text { Impact of Emotional } \\
\text { Stress }\end{array}$ & Relax Every Day & Selected Websites \\
\hline & $\begin{array}{c}\text { No Family } \\
\text { Support }\end{array}$ & Theory A & $\begin{array}{l}\text { Take Responsibility(Serenity } \\
\text { Prayer) }\end{array}$ & Custom meaningful sounds and backgrounds \\
\hline & & Theory B & $\begin{array}{l}\text { Re-examine your values and live } \\
\text { by them }\end{array}$ & Personalized Video Creation: Humor \\
\hline & & Theory C & 24/7 consultation available & Video Conference \\
\hline & & & Remote Monitoring & E-Evaluation Survey \\
\hline
\end{tabular}

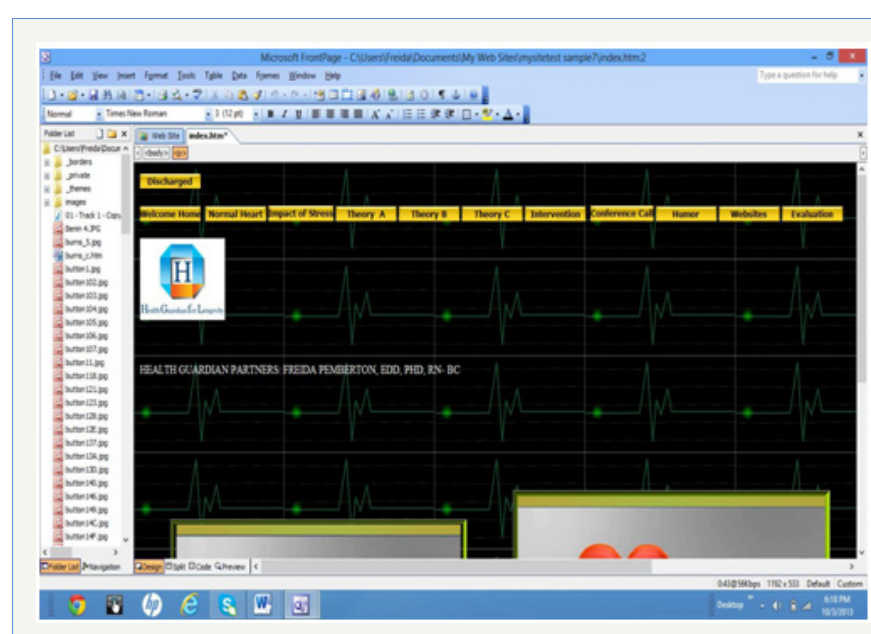

Figure 1:

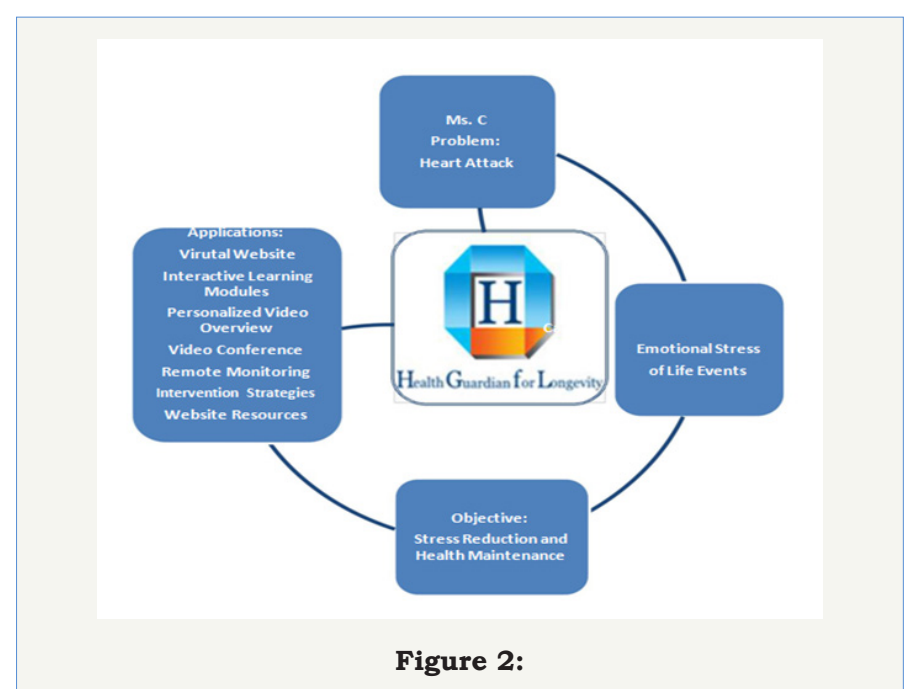

The patient-clients who were given access to the program website and their own personalized care-management page designed specifically reported that it was extremely helpful, and passed the information to others. Their unsolicited testimony resulted in a flood of calls to our department from individuals interested in participating. Both the positive response from participants and subsequent interest from those outside our study strongly suggest health care consumers' openness to a different, more integrated approach to healthcare management using the types of technology most people use every day Figure 1 and Figure 2.

\section{Summation}

The Agency for Healthcare Research and Quality [7] points out, though the consensus of research has shown that patient outcomes are better when patients and their families are included in the decision-making process and the management of their care, and when personal preferences and cultural differences are addressed in the context of health management, there continues to be a delay in incorporating patient-centered care concepts in the everyday decision-making process.

The Health Guardian for Longevity program seeks to address this shortcoming by delivering an interactive, web-based custom health promotion program that will foster self-sufficiency for the patient-client, their family and the community, encouraging healthier long-term lifestyle behaviors, and reducing the frequency and likelihood of return visits for future health events. As we collect data in the forthcoming case studies on the impact of the Health Guardian for Longevity program as an innovative, progressive approach to tailored healthcare practice, we will make the necessary adjustments to enhance positive patient outcomes.

\section{References}

1. Kreuter M, Strecher B, Glassma B (1999) One size does not fit all: The Society of Behavioral Medicine. Ann Behav Med 21(4): 276-283.

2. Pennsylvania State University (1992) The report of the task force on distance education. University Park, USA.

3. (2011) Vital Statistics Data of the New York State Department of HealthBureau of Biometrics and Health Statistics, as of Leading causes of death 2000-2009. 
4. Skinner CS, Siegfried JC, Kegler MC, Strecher VJ (1993) The potential of computer in patient education. Patient Educ Couns 22(1): 27-34.

5. Gustafson, Hawkins, Boberg, Pingree, Serlin, Graziano, et al. (1999) Impact of a patient-centered, computer-based health information/ support system. Am Jof Prev Med 16(1): 1-9.

6. DiMarco C, Wiljer D, Hovy E (2007) Self-managed access to personalized healthcare through automated generation of tailored health educational material from electronic health records. Virtual Healthcare Interaction: Paper from the AAAI Fall Symposium pp. 1-7.

7. Agency for Healthcare Research and Quality national Institute of Mental Health (2001) Patient-centered care: Customizing care to meet patients needs. Agency for Healthcare Research and Quality National Institute of Mental Health, USA. 